\title{
Multi-objective Robust Optimization to Solve Energy Scheduling in Buildings Under Uncertainty
}

\author{
Joao Soares, Zita Vale, Nuno Borges \\ GECAD - Knowledge Engineering and \\ Decision-Support Research Center \\ Polytechnic of Porto (ISEP/IPP), Portugal \\ \{joaps, zav, ndsbs\}@isep.ipp.pt
}

\author{
Fernando Lezama \\ Computer Science Department \\ Instituto Nacional de Astrofísica, Óptica \\ y Electrónica (INAOE), Mexico \\ f.lezama.mx@ieee.org
}

\author{
Nelson Kagan \\ ENERQ - Centro de Estudos em \\ Regulação e Qualidade de Energia \\ USP - University of São Paulo - \\ Polytechnic School, Brazil \\ nelsonk@pea.usp.br
}

\begin{abstract}
With the high penetration of renewable generation in Smart Grids (SG), the uncertainty behavior associated with the forecast of weather conditions possesses a new degree of complexity in the Energy Resource Management (ERM) problem. In this paper, a Multi-Objective Particle Swarm Optimization (MOPSO) methodology is proposed to solve ERM problem in buildings with penetration of Distributed Generation (DG) and Electric Vehicles (EVs) and considering the uncertainty of photovoltaic (PV) generation. The proposed methodology aims to maximize profits while minimizing $\mathrm{CO} 2$ emissions. The uncertainty of PV generation is modeled with the use of Monte Carlo simulation in the evaluation process of the MOPSO core. Also, a robust optimization approach is adopted to select the best solution for the worst-case scenario of PV generation. A case study is presented using a real building facility from Brazil, to verify the effectiveness of the implemented robust MOPSO.
\end{abstract}

Index Terms-- Energy Resources Management, $\mathrm{CO}_{2}$ Emissions, Multi-Objective Particle Swarm Optimization, Robust Optimization.

\section{INTRODUCTION}

Modern power networks adopting Smart Grid (SG) technologies are leading the modifications of operation and management of resources worldwide. Such networks, with the capability of integrating distributed energy resources (DER), such as loads, generator units, Energy Storage Systems (ESSs) and Electric Vehicles (EVs), possess a new degree of complexity in operation and management [1].

One positive aspect of SG technologies is the increasing penetration of renewable energy production, such as the wind and photovoltaic, that contributes positively to the reduction of the carbon footprint. However, in contrast to conventional generation units, renewable sources are characterized by a high degree of uncertainty and variability. Given this degree of uncertainties, it is important to use advanced tools, using robust control and predictive models, to handle with such stochastic and uncertain behavior. Robust optimization has proved to be a promising method to deal with the uncertainties in the optimization problems [2].

Several studies to solve the energy resources management (ERM) problem in SGs under uncertainty have been reported in the recent literature. For instance, in [3], in the context of smart homes, robust optimization is used to model the problem input uncertainties concerning the production of a photovoltaic system with the primary objective of performing the scheduling of various types of electrical charges. In [4], the authors evaluate Demand Response (DR) programs based on real-time prices for the management of residential loads through two approaches, namely stochastic optimization, and robust optimization. Stochastic optimization adopts the scenariobased approach via Monte Carlo (MC) simulation for minimizing the expected electricity payment for the entire day, while controlling the financial risks associated with real-time electricity price under uncertainties via the conventional downside risks formulation. Price change intervals are considered in the robust optimization for minimizing the worstcase electricity payment. Both approaches are formulated through a mixed integer linear programming (MILP). Reference [5] presents an energy management system of a building under the influence of multiple sources of uncertainty, such as the energy demand level concerning carbon emissions, primary energy savings, and the prices (prices of fuel and electricity tariffs). The optimization is then modeled as a multiobjective problem for a commercial building aiming to minimize costs and energy consumption simultaneously and adopts robust optimization to overcome the uncertainties mentioned above. In [6], a framework capable of performing power management in a microgrid based on a robust optimization is developed. Various types of uncertainty are considered, such as the production based on variable renewable sources, the cost of production and the energy consumption. The uncertainty is represented by prediction intervals, estimated by a neural network and by the Non-dominated Sorting Genetic Algorithm (NSGA -II) to obtain the upper and 
lower limits, representing a range of values where it is expected that the parameter with uncertainty values tend to match, with a certain confidence level. In [7], it is presented a method to deal with the optimization of energy management for the production and consumption of a microgrid connected to the network and incorporating renewable production technologies. A robust formulation was used to address the uncertainty in renewable energy sources production units. It is worth noting that in the current literature, the subject of robust optimization applied to meta-heuristics is not well exploited since robust models are usually converted to linear and deterministic optimization. However, in situations with a large number of variables or considering non-linear models, the deterministic approach may be impractical.

This paper presents the application of Multi-Objective Particle Swarm Optimization (MOPSO) to solve the non-linear ERM problem in one building, with penetration of DG and EVs, and adopting robust optimization under uncertainty. The proposed methodology considers a multi-objective approach that aims to maximize the profit while minimizing $\mathrm{CO}_{2}$ emissions. Besides, the method considers uncertainty associated with the production of electricity by photovoltaic energy sources. Such uncertainty is modeled with the use of MC simulation. Moreover, the robust model developed in this paper is based on [2]. A case study is presented using a real building facility in Brazil from the University of Sao Paulo, with DG, EVs, and ESS, to verify the effectiveness of the robust algorithm implemented. Results suggest that robust optimization can be performed actually inside the traditional framework of MOPSO, leading to efficient Pareto fronts.

\section{PROBLEM FORMULATION}

In this section, the mathematical model and uncertainty consideration is presented.

\section{A. Mathematical model}

The envisaged problem is a hard combinatorial MixedInteger Non-Linear Programming (MINLP) problem due to a large number of continuous and discrete variables. The two conflicting objectives of the building management are to maximize profits (Eq. (1)) while minimizing $\mathrm{CO}_{2}$ emissions (Eq. (2)).

Profits then are modeled as the difference between revenues and operational costs, such as:

$$
\begin{aligned}
& \text { Maximize } f_{1}=[R-C] \\
& R=\sum_{t=1}^{T}\left[\left(\sum_{v=1}^{N_{v}} P_{\text {charg }}^{v}(t) \cdot c_{\text {charg }}^{v}(t)\right)+r_{p p}\right] \\
& C=\sum_{t=1}^{T}\left[\left(\sum_{d=1}^{N_{d g}} P_{d g}^{d}(t) \cdot c_{d g}^{d}(t)+\sum_{s=1}^{N_{v}} P_{s p}^{s}(t) \cdot c_{s p}^{s}(t)\right)\right]
\end{aligned}
$$

where $R$ is the building revenue (m.u.) and $C$ represents the cost of the resources managed by the building (also in m.u.). For function $R, v$ is the index for EVs. The parameters are described by: $N_{v}$ is the number of EVs; $c_{\text {charg }}^{v}(t)$ is the price for the charging process of EV $v$ in period $t$ (m.u.); $r_{p p}$ is the incentive for achieving the peak power value. The variables are described by: $P_{\text {charg }}^{v}(t)$ is the active power charge of EV $v$ in period $t(\mathrm{~kW})$.

In function $C$ (Costs), the indices are represented by: $d$ is an index of DG units; $s$ is an index of external suppliers. The parameters are described by: $N_{d g}$ is the number of DG units; $N_{s p}$ is the number of external electricity suppliers; $c_{d g}^{d}(t)$ is the generation price of DG unit $d$ in period $t$ (m.u.); $c_{s p}^{s}(t)$ is the energy price of external supplier $s$ in period $t$ (m.u.); $c_{\text {disch }}^{v}(t)$ is the discharging cost of EV $v$ in period $t$ (m.u.); $c_{\text {charg }}^{s s}(t)$ is the price for the charging process of ESS $s s$ in period $t$ (m.u.). The variables are described by: $P_{d g}^{d}(t)$ is the active power generation of DG unit $d$ in period $t(\mathrm{~kW}) ; P_{s p}^{s}(t)$ is the active power generation of the external supplier $s$ in period $t(\mathrm{~kW})$; $P_{\text {disch }}^{v}(t)$ is the active power discharge of EV $v$ in period $t$ $(\mathrm{kW}) ; P_{\text {disch }}^{S S}(t)$ is the active power discharge of ESS $s s$ in period $t(\mathrm{~kW})$.

On the other hand, $\mathrm{CO}_{2}$ emissions of the building are modeled as:

$$
\begin{aligned}
& \text { Minimize } f_{2}= \\
& \sum_{t=1}^{T}\left[\left(\sum_{\Omega_{d g}^{d}} P_{d g}^{d}(t) \cdot E_{d g}^{d}(t)+\sum_{\Omega_{s p}^{s}} P_{s p}^{s}(t) \cdot E_{s p}^{s}(t)\right)\right]
\end{aligned}
$$

where $\Omega_{d g}^{d}$ is a set of DG units with $\mathrm{CO}_{2}$ emissions; $\Omega_{s p}^{s}$ is a set of external suppliers with $\mathrm{CO}_{2}$ emissions. The parameters are described by: $E_{d g}^{d}(t)$ is the $\mathrm{CO}_{2}$ emissions of DG unit $d$ in period $t\left(\mathrm{kgCO}_{2} / \mathrm{kWh}\right) ; E_{s p}^{s}(t)$ is the $\mathrm{CO}_{2}$ emissions of external supplier $s$ in period $t\left(\mathrm{kgCO}_{2} / \mathrm{kWh}\right)$.

Some constraints of this problem can be found in [8], such as EV charging and discharging rates, battery capacity and balance considering predicted demand and location, technical limits of ESSs, balance, and capacity in each period, dispatchable DG capacity and supplier's limits. In addition, an innovative DR model which considers a daily peak power pricing $\left(c_{\text {peakPower }}\right)$ and an incentive to minimize it $\left(r_{\text {peakPower }}\right)$ is proposed. The daily peak power pricing depends on the peak power value $\left(P_{\text {peak }}\right)$, that represents the maximum energy supplied by the external supplier for the entire day. The $c_{\text {peakPower }}$ can be calculated as:

$$
\begin{aligned}
& c_{\text {peakPower }=}= \\
& \begin{cases}p_{\text {level }}(1), & \text { if } \lim _{\min }(1) \leq p_{\text {peak }} \leq \lim _{\max }(1) \\
p_{\text {level }}(2), & \text { if } \lim _{\min }(2) \leq p_{\text {peak }} \leq \lim _{\max }(2) \\
\vdots & \\
p_{\text {level }}(n), & \text { if } \lim _{\min }(n) \leq p_{\text {peak }} \leq \lim _{\max }(n)\end{cases}
\end{aligned}
$$

where the parameters are described by: $\lim _{\min }$ is the minimum power limit; lim $_{\max }$ is the maximum power limit; $p_{\text {level }}$ is the peak power price by level. 
The $r_{\text {peakPower }}$ is paid if the $P_{\text {peak }}$ of the building does not exceed $200 \%$ of the average daily demand $\left(P_{\text {mean }}\right)$ as:

$$
r_{\text {peakPower }}=\left\{\begin{array}{l}
\frac{c_{\text {peak }}}{2}, \text { if } P_{\text {peak }} \leq 2 P_{\text {mean }} \\
0, \text { if } P_{\text {peak }}>2 P_{\text {mean }}
\end{array}\right.
$$

\section{B. Uncertainties}

With the high penetration of renewables, weather forecast became critical to the vast majority of electricity players. However, it also constitutes imperfect information due to forecasting errors. Certain input parameters in the proposed optimization model are not deterministic, i.e. the wind and photovoltaic production, and those inputs can change significantly after the optimization process. In day-ahead forecast with large time horizon, these variables affect the value of objective function due to the restriction of power balance as follows:

$$
\begin{aligned}
& \sum_{d=1}^{N_{d g}} P_{d g}^{d}(t)+\sum_{s=1}^{N_{s p}} P_{s p}^{s}(t)+\sum_{v=1}^{N_{v}}\left(P_{\text {disch }}^{v}(t)-P_{\text {charg }}^{v}(t)\right) \\
& +\sum_{s s=1}^{N_{s s}}\left(P_{\text {disch }}^{s s}(t)-P_{\text {charg }}^{s s}(t)\right)+\sum_{a=1}^{N_{p v}}\left(P_{p v}^{a}(t)+e_{p v}^{a}(t)\right) \\
& =\sum_{l=1}^{N_{l}} P_{\text {load }}^{l}(t), \forall t
\end{aligned}
$$

where $P_{p v}^{a}(t)$ is the forecasted power generation of PV $a$ (i.e., solar generations) at time $t$, and $e_{p v}^{a}(t)$ is an error associated with the forecast of the solar conditions. This error represents then the uncertainty associated with PV generation and modifies the value of such variable depending on the scenario considered.

There are a few methods that can be deployed to handle uncertainties in this type of inputs. In this paper, a robust optimization approach is used for the analysis of the worst-case scenario. A solution may be called robust if, despite the variation of the inputs under uncertainty, it remains feasible and the objective function value changes only slightly. In other words, if the uncertainty inputs fall within a predefined range, then the optimization procedure always search for a solution that gives the better payoff in the best worst-case situation.

Forecast and associated errors are assumed based on previous experiences, trying to simulate real-world behavior. In this paper, the stochastic model is used assuming that a correct set of scenarios can be generated considering future availability of such historical data. In fact, scenario generation is a broad topic that is beyond the scope of this paper. Nevertheless, in the current literature some authors have presented approaches than can be implemented in scenario generation tools in control centers for the ERM. For instance, In [9] authors use Monte Carlo Simulation (MCS) to capture the uncertainty of the wind power forecast. A scenario reduction technique is then used to reduce the number of generated scenarios. Furthermore, they assume that solar forecast errors follow a normal distribution. As in [9], in this paper we generate 10 independent scenarios based on solar forecast, which results in ten scenarios with an equal probability of 0.1 .

\section{Multi ObJective PARTICLE SWARM Optimization (MOPSO)}

MOPSO is an advanced optimization algorithm to solve multi-objective problems [10]. MOPSO adopts an external repository and uses a mutation operator aiming to explore the remote regions of the search space and the full range of each decision variable. In this paper, we employ mutation of the strategic parameters as used in Evolutionary PSO (EPSO) [11] instead of the usually fixed parameters of the original MOPSO. Therefore, two types of mutation occur during the search loop, namely mutation of the parameters of the velocity equation and mutation in the position of some particles (randomly selected). The proposed methodology uses three strategic parameters, namely: inertia, memory, and cooperation. At the beginning of the process, the values of these weights are randomly generated between 0 and 1 . After that, the weights are changed in each iteration using a Gaussian mutation distribution according to:

$$
W_{i}^{g+1}=W_{i}^{g}+\delta \cdot N(0,1)
$$

where $W_{i}^{g+1}$ is the new mutated weight of particle $i, W_{i}^{g}$ represents the weight of particle $i$, and $\delta$ is a learning parameter with a range between 0 and 1 . A high value of $\delta$ adds more importance to mutation, whereas $N(0,1)$ is a random number following a normal distribution with mean 0 and variance equal to 1 . The strategic parameters are limited to values between 0 and 1 at this stage. This modification improves the convergence rate and the overall front of non-dominated solutions since higher exploratory properties are introduced in the search process.

With the calculation of these weights, the new particle velocity is calculated as:

$$
\begin{aligned}
& V_{i}^{g+1}= \\
& W_{i(\text { ine })}^{g} \cdot V_{i}^{g}+W_{i(\text { mem })}^{g} \cdot\left(x_{\text {pbest }}-X_{i}^{g}\right)+W_{i(\text { coop })}^{g} \cdot\left(x_{\text {gbest }}-X_{i}^{g}\right)
\end{aligned}
$$

where $x_{\text {pbest }}$ is the best past experience of particle $i ; x_{g b e s t}$ is the best global experience of all the particles; $V_{i}^{g}$ is the velocity of particle $i$ in iteration $g ; V_{i}^{g+1}$ is the new calculated velocity of particle $i ; X_{i}^{g}$ is the position of particle $i$ in iteration $g$; $W_{i(\text { ine })}^{g}$ is the inertia weight component of particle $i$ in iteration $g ; W_{i(m e m)}^{g}$ is the memory weight component of particle $i$ in iteration $g ; W_{i(\text { coop })}^{g}$ is the cooperation weight component of particle $\underline{i}$ in iteration $g$.

After the calculation of the new velocity for each particle, the position is updated according to:

$$
X_{i}^{g+1}=X_{i}^{g}+V_{i}^{g+1}
$$


The algorithm stops after a defined number of iterations, or a custom condition is reached. This setup is widely used in other multi-objective metaheuristic algorithms [12].

The main factor of innovation presented in this model focuses on the consideration of the uncertainties regarding production from photovoltaic panels and wind turbine. To handle such uncertainties, our MOPSO algorithm adopts a robust optimization approach that focuses on finding the best solutions for the worst-case scenario.

To do that, during the evaluation process of each particle, the variables under uncertainty are perturbated and a set of different scenarios are generated using MCS. Then, for each scenario, the particle is evaluated in the multi-objective space (Eq. (1) and Eq. (2)) and, through a variable that decides which objective the user wants to give more relevance, the worst-case solution is chosen (i.e., a robust solution). This process is repeated for each particle in the swarm. After that, the nondominated solutions are updated using the robust solutions found during the evaluation process. At the end of each iteration, the non-dominated particles are stored in a repository to generate the Pareto front. The entire cycle is repeated until a defined number of iterations is reached. A flow chart of MOPSO is presented in Fig. 1.

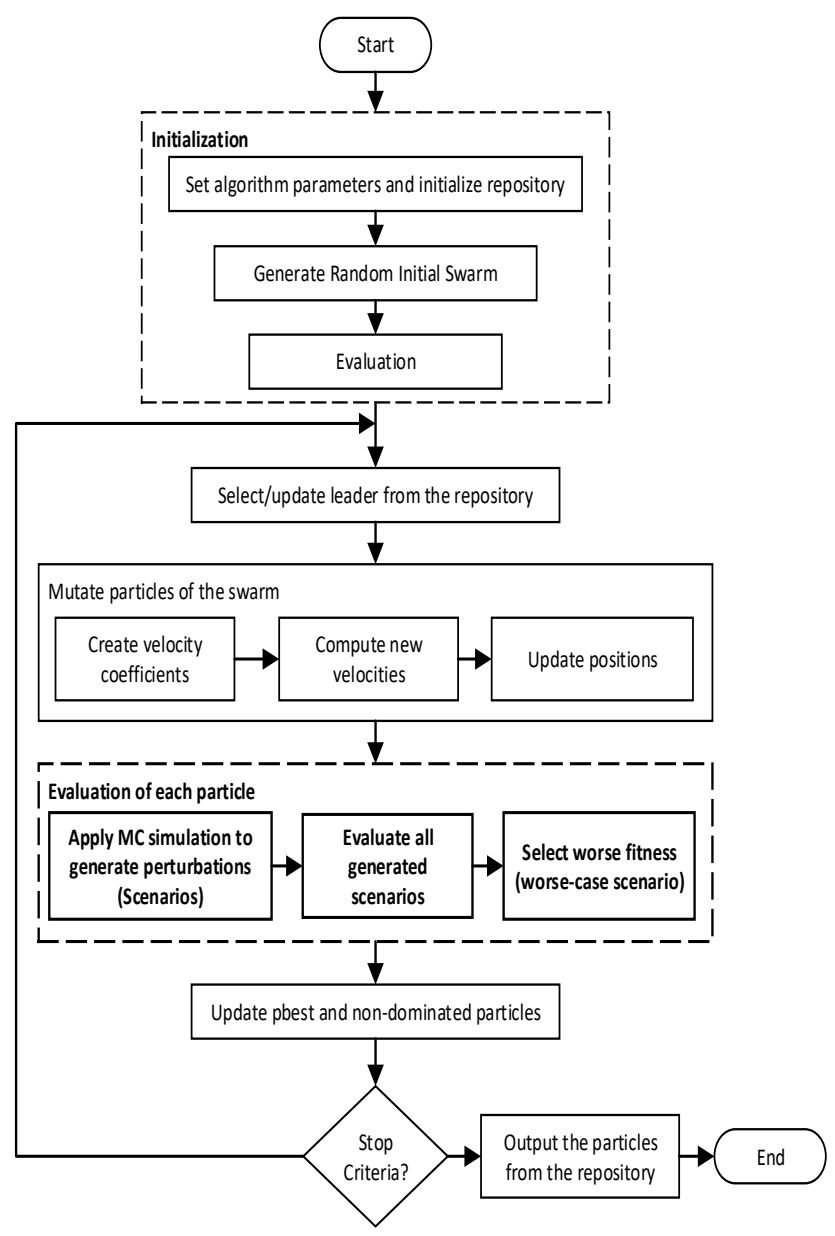

Figure 1. Flowchart of the developed MOPSO

As different metaheuristic-based algorithms, MOPSO has some control parameters that modify the performance of the algorithm in some case studies. In this work, a suitable set of parameters was obtained by empirical experimentation and by previous recommendations from the literature [10]. Those parameters are summarized in Table I. The repository size was set to 100 , as suggested in the literature, to obtain a proper Pareto front.

TABLE I. MOPSO PARAMETERS

\begin{tabular}{|c|c|c|}
\hline \multicolumn{2}{|c|}{ Parameter } & Description \\
\hline \multicolumn{2}{|c|}{ Number of particles } & 10 \\
\hline \multicolumn{2}{|c|}{ Repository size } & 100 \\
\hline \multicolumn{2}{|c|}{ Inertia Weight } & \\
\hline \multicolumn{2}{|c|}{$\begin{array}{l}\text { Acceleration Coefficient } \\
\text { Best Position } \\
\end{array}$} & $\begin{array}{l}\text { Gaussian mutation weights } \\
\text { (initial weights randomly generated }\end{array}$ \\
\hline \multicolumn{2}{|c|}{ Cooperation Coefficient } & \\
\hline \multicolumn{2}{|c|}{ Perturbation Coefficient } & \\
\hline \multicolumn{2}{|c|}{$\begin{array}{l}\text { Mutation learning } \\
\text { parameter }(\delta)\end{array}$} & 0.20 \\
\hline \multicolumn{2}{|c|}{ Number of divisions } & 30 \\
\hline \multicolumn{2}{|c|}{ Initial swarm population } & $\begin{array}{l}\text { Randomly generated between the upper } \\
\text { and lower bounds of variables }\end{array}$ \\
\hline \multicolumn{2}{|c|}{ Mutation rate of particles } & 0.50 \\
\hline \multicolumn{2}{|c|}{ Mutation dimensions } & Random $10 \%$ dimensions \\
\hline \multicolumn{2}{|c|}{$\begin{array}{l}\text { Velocity clamping factor } \\
\qquad\left(C_{\text {factor }}\right)\end{array}$} & 1 \\
\hline $\begin{array}{l}\text { Stopping } \\
\text { Criteria }\end{array}$ & Max. & 2000 iterations (cycles) \\
\hline \multicolumn{2}{|c|}{ Max. Positions $\left(x_{\max }\right)$} & $\begin{array}{l}\text { Equal to the upper bounds of the } \\
\text { variables }\end{array}$ \\
\hline \multicolumn{2}{|c|}{ Min. Positions $\left(x_{\min }\right)$} & $\begin{array}{c}\text { Equal to the lower bounds of the } \\
\text { variables }\end{array}$ \\
\hline \multicolumn{2}{|c|}{ Max. Velocities $\left(v_{\max }\right)$} & $\frac{x_{\max }-x_{\min }}{2} \cdot C_{\text {factor }}$ \\
\hline \multicolumn{2}{|c|}{ Min. Velocities $\left(v_{\min }\right)$} & $-v_{\max }$ \\
\hline
\end{tabular}

\section{RESULTS AND DISCUSSION}

A case study on a real building, in São Paulo, Brazil, namely the ENERQ building from USP - Polytechnic School, is used to show the effectiveness of the proposed methodology. The building can manage $1 \mathrm{PV}$ unit, 4 external suppliers, 1 storage unit and 3 EVs. Table II shows the energy resources of the considered case study regarding the information of price in monetary units per kWh (m.u./kWh) and availability in kW.

TABLE II. ENERGY RESOURCE DATA

\begin{tabular}{|c|c|c|c|c|}
\hline \multirow{2}{*}{\multicolumn{2}{|c|}{ Energy resources }} & $\begin{array}{c}\text { Availability } \\
(\mathbf{k W})\end{array}$ & $\begin{array}{c}\text { Prices } \\
\text { (m.u./kWh) }\end{array}$ & \multirow[t]{2}{*}{ Units } \\
\hline & & $\min -\max$ & $\min -\max$ & \\
\hline \multicolumn{2}{|c|}{ Photovoltaic } & $0-5.00$ & 0.00 & 1 \\
\hline \multicolumn{2}{|c|}{ External Supplier } & $0-15.00$ & $0.07-0.32$ & 4 \\
\hline \multirow{2}{*}{ Storage } & Charge & $0-10.00$ & 0.00 & \multirow{2}{*}{1} \\
\hline & Discharge & $0-10.00$ & 0.00 & \\
\hline \multirow{2}{*}{$\begin{array}{l}\text { Electric } \\
\text { Vehicle } \\
\end{array}$} & Charge & $0-9.00$ & 0.13 & \multirow{2}{*}{3} \\
\hline & Discharge & $0-9.00$ & 0.15 & \\
\hline \multicolumn{2}{|l|}{ Load } & $1.33-7.61$ & 0.00 & 1 \\
\hline
\end{tabular}


The external suppliers were selected with different emission rates and energy prices. Table III presents the different types of external suppliers used in this case study based on four European countries (Portugal, Spain, Germany and France) [13]-[17].

TABLE III. EXTERNAL SUPPLIERS DATA

\begin{tabular}{c|c|c|c}
\hline \hline Scenario & $\begin{array}{c}\mathbf{C O}_{2} \text { emissions } \\
\left(\mathbf{k g C O}_{2} / \mathbf{k W h}\right)\end{array}$ & $\begin{array}{c}\text { Prices } \\
(\mathbf{m . u} . / \mathbf{k W h})\end{array}$ & Tariff type \\
\hline \hline Portugal & 0.23 & $0.0927-0.3177$ & Tri tariff \\
\hline Spain & 0.25 & $0.0742-0.0993$ & Hourly tariff \\
\hline France & 0.07 & $0.1150-0.1636$ & Bi tariff \\
\hline Germany & 0.35 & 0.2013 & Simple tariff \\
\hline \hline
\end{tabular}

The parameters used for the DR model are shown in Table IV. The incentive monetary value is equal to half of the amount paid for the power peak value.

TABLE IV. LEVELS OF PEAK POWER PRICES

\begin{tabular}{c|c}
\hline \hline Power Levels (kW) & Price (m.u.) \\
\hline \hline $1-3$ & 0.05 \\
\hline $4-6$ & 0.06 \\
\hline $7-9$ & 0.07 \\
\hline $10-12$ & 0.08 \\
\hline $13-15$ & 0.09 \\
\hline \hline
\end{tabular}

The experiments were done using MATLAB 2014b 64 bits in a PC with an Intel Xeon E5-2620 processor and 16 GB of RAM running Windows 10. For comparison purposes, a random selection scenario rather than the worst-case scenario selection in the evaluation process was also implemented. The reported results correspond to the average over 30 runs of MOPSO.

Figure 2 shows the Pareto front obtained with the robust approach and with the random approach.

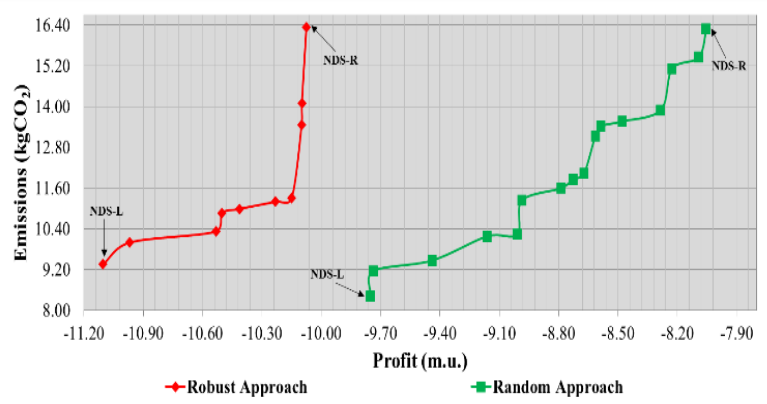

Figure 2. Pareto front for robust and random approach

The number of non-dominated solutions (NDS) obtained on average was 10 and 16 NDS for the robust and random approach respectively. Figure 2 also show the best solution regarding Emissions (i.e., NDS-L) and Profit (i.e., NDS-R) of both Pareto fronts. From the point of view of the uncertainty modeling, the robust approach is a conservative Pareto front since represent the best solutions under the effect of the worsecase scenario. On the other hand, despite the better quality of the Pareto front of the random approach, by not considering the worst-case scenarios those solutions represent unprotected solutions from the uncertainties in this problem.

Table V shows the selected NDS from the Pareto curve. It is worth noting that, for this particular case study, the PV unit is not sufficient to feed the total consumption, resulting in negative profits (i.e., operational costs higher than profits).

TABLE V. SELECTED NON-DOMINATED SOLUTIONS

\begin{tabular}{c|c|c|c|c}
\cline { 2 - 5 } & \multicolumn{2}{|c|}{ Robust } & \multicolumn{2}{c}{ Random } \\
\hline NDS & L & R & L & R \\
\hline \hline Profit (m.u) & -11.103 & -10.075 & -9.751 & -8.055 \\
\hline $\mathbf{C O}_{\mathbf{2}}(\mathbf{k g})$ & 9.356 & 16.338 & 8.416 & 16.287 \\
\hline \hline
\end{tabular}

In a multi-objective formulation, the algorithm returns a set of solutions rather than a specific one (i.e., the Pareto front). The expert (e.g., network operator) then should decide to select a specific solution based on the trade-off of the objectives involved in the optimization. In this case study, NDS-L represents the solution with less $\mathrm{CO}_{2}$ emissions (i.e., the best value of Eq. (2)) and high cost (worst value of Eq. (2)). On the contrary, NDS-R shows the most efficient solution in terms of costs and worst $\mathrm{CO}_{2}$ emissions front the Pareto front.

To analyze the scheduling of a particular solution, Figs. 3 and 4 depict the energy scheduling result for both, the robust and random approaches, of a selected NDS-L. It can be noticed that the storage discharge is a resource used in both solutions. This is due to the fact that it is a resource that does not involve any cost to the building (i.e., $c_{c h a r g}^{S S}=0$ ), since the storages are considered the property of the building. The opportunity to use the EV discharge energy is only possible between $8 \mathrm{~h}$ and $20 \mathrm{~h}$, since the case study represents an office building. Moreover, EVs discharge feature is usually used in periods when external supplier prices are higher than the incentive that the building can provide to EVs for their energy, i.e., periods 14-to-17 in the robust approach and periods 10-to17 in the random approach. Overall, the resources such as the EVs and ESSs discharge are commonly used to decrease the energy provided by external suppliers at higher costs.

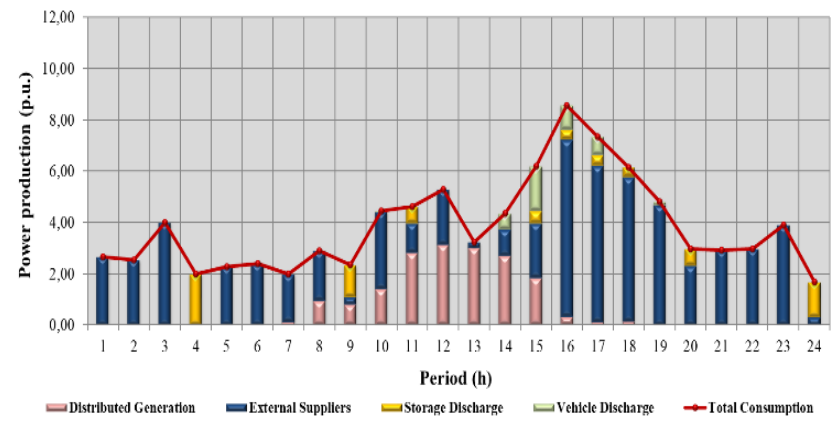

Figure 3. Optimal resource scheduling of NDS-L for the robust approach. 


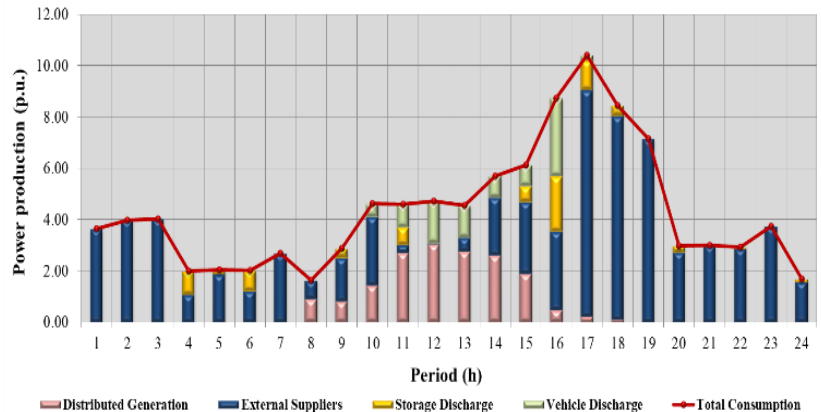

Figure 4. Optimal resource scheduling of NDS-L for the random approach.

Finally, Fig.5 shows the total energy consumption for NDS-L solutions obtained with the robust and random approaches.

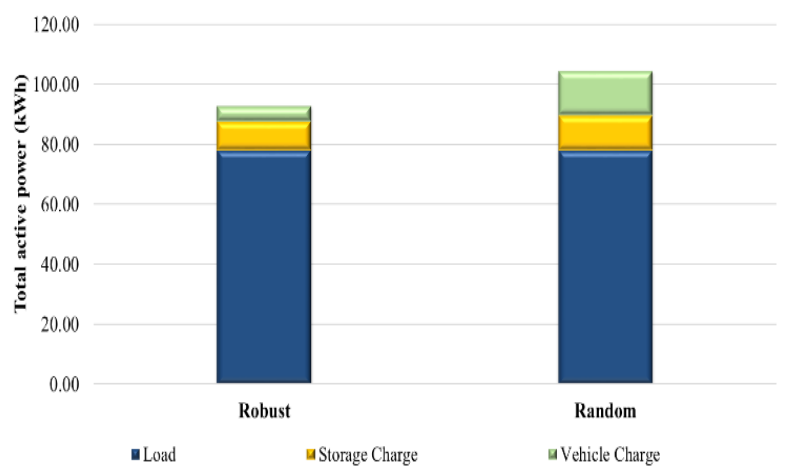

Figure 5. Energy consumption of NDS-L for the robust and random approach.

It can be seen that the EV charge feature is higher in the random approach. This behavior was expected since in this solution exists a major use of EVs discharge feature, leading to an additional EVs energy necessity. The EVs charge is made mainly in the afternoon periods, so the owners guarantee a minimum battery requirement to make the return trip to home. Besides, the charging of EVs is still performed during periods of high DG production (12h-14h). In the presence of uncertainties, if a worst-case scenario occurs, it is probable that the scheduling cannot fulfill the minimum energy required to make the trip back home. In such cases, a robust solution that guarantees a higher level of energy (even at a higher price) is preferred. Regarding the ESS charging, this is carried out in periods when the building can buy energy from external suppliers at lower prices, corresponding essentially to periods during the night (23h-8h). Moreover, as in the case of EVs, the ESS charge is commonly performed during periods of high DG production (12h-14h).

\section{CONCLUSION}

This paper presented a method for intelligent energy management of a building using MOPSO to maximize the profit and minimize the $\mathrm{CO}_{2}$ emissions. MOPSO is responsible for finding a set of non-dominated solutions, i.e., the Pareto front. Also, a robust optimization mechanism is incorporated in the evaluation process to search for solutions that represent the optimal decisions in a given worst-case scenario. The robust optimization model developed in this work can be useful to obtain a quick solution for the day-ahead energy resource management, allowing the building operator to solve the problem with a more conservative view regarding uncertainties of photovoltaic generations. As future work, more sources of uncertainties, like the ones associated with EVs behavior, can be incorporated in the robust approach. Adding more uncertainties to the model will also increase the complexity and execution time of the algorithm (i.e., more $\mathrm{MC}$ simulations will be required to evaluate a solution in the core of MOPSO). Therefore, efficient scenarios' reduction techniques or stochastic optimization are other interesting venues of research.

\section{ACKNOWLEDGMENT}

This work has received funding from the Project NetEffiCity (ANI|P2020 18015), and from FEDER Funds through COMPETE program and from National Funds through FCT under the project UID/EEA/00760/2013.

\section{REFERENCES}

F. Lezama, J. Palominos, A. Y. Rodriguez-Gonzalez, A. Farinelli, and E. Munoz de Cote, "Agent-Based Microgrid Scheduling: An ICT Perspective," Mob. Networks Appl., May 2017.

T. by Robert Marijt and profdrir J. Hensen, "Multi-objective Robust Optimization Algorithms for Improving Energy Consumption and Thermal Comfort of Buildings," 2009.

C. Wang, Y. Zhou, B. Jiao, Y. Wang, W. Liu, and D. Wang, "Robust optimization for load scheduling of a smart home with photovoltaic system," Energy Convers. Manag., vol. 102, pp. 247-257, 2015.

Z. Chen, L. Wu, and Y. Fu, "Real-Time Price-Based Demand Response Management for Residential Appliances via Stochastic Optimization and Robust Optimization," IEEE Trans. Smart Grid, vol. 3, no. 4, pp. 1822-1831, Dec. 2012.

K. Akbari, M. M. Nasiri, F. Jolai, and S. F. Ghaderi, "Optimal investment and unit sizing of distributed energy systems under uncertainty: A robust optimization approach," Energy Build., vol. 85, pp. 275-286, Dec. 2014.

E. Kuznetsova, C. Ruiz, Y.-F. Li, and E. Zio, "Analysis of robust optimization for decentralized microgrid energy management under uncertainty," Int. J. Electr. Power Energy Syst., vol. 64, pp. 815832, Jan. 2015.

Y. Zhang, N. Gatsis, and G. B. Giannakis, "Robust Energy Management for Microgrids With High-Penetration Renewables," IEEE Trans. Sustain. Energy, vol. 4, no. 4, pp. 944-953, Oct. 2013. J. Soares, N. Borges, Z. Vale, and P. B. Oliveira, "Enhanced MultiObjective Energy Optimization by a Signaling Method," Energies, vol. 9, no. 10, p. 807, 2016

W. Su, J. Wang, and J. Roh, "Stochastic Energy Scheduling in Microgrids With Intermittent Renewable Energy Resources," IEEE Trans. Smart Grid, vol. 5, no. 4, pp. 1876-1883, Jul. 2014.

C. A. C. Coello, G. T. Pulido, and M. S. Lechuga, "Handling multiple objectives with particle swarm optimization," Evol. Comput. IEEE Trans., vol. 8, no. 3, pp. 256-279, 2004.

V. Miranda, H. Keko, and A. Jaramillo, "EPSO: Evolutionary particle swarms," Stud. Comput. Intell., vol. 66, pp. 139-167, 2007. F. Lezama, A. Y. Rodriguez-Gonzalez, and E. M. de Cote, "Load pattern clustering using differential evolution with Pareto Tournament," in IEEE Congress on Evolutionary Computation (CEC), 2016, pp. 241-248.

Red Eléctrica de España, “Active Energy Invoicing Price,” 2016. .

ERSE, "Preços de referência no mercado liberalizado de energia elétrica e gás natural em portugal continental," 2016.

E.ON, "Finden Sie lhr optimales Stromprodukt," 2016.

EDF, "Grilles tarifaires de l'offre de fourniture d'électricité," 2016. European Commission, "The 2013 EU Reference scenario: EU energy , transport and GHG emissions trends to 2050," 2014. 